\title{
Relaxation of Polyvinyl Butyral Film with non-uniform Thickness
}

\author{
Aleš Mareška (0000-0003-1613-6626), Tereza Kordová (0000-0002-9325-9593), Martin Havlík Míka (0000-0003- \\ 2472-3628) \\ Faculty of Chemistry and Technology, University of Chemistry and Technology, Prague. Technická 5, Dejvice, \\ 16628 Praha 6. Czech Republic. E-mail: mareskaa@vscht.cz; kordovat@vscht.cz; kordovat@vscht.cz
}

Polyvinyl butyral is a material that is widely used in the manufacture of laminated glass sheets. Polyvinyl butyral film is widely used as an intermediate layer for laminated automotive or architectural glass. The intermediate layer primarily ensures the safety function of the laminated glass. Other advantages of laminated glass with a polyvinyl butyral interlayer include that it can be adapted to perform other functions, including acoustic functions, anti-reflection functions or functions enabling information to be projected on the glass with this interlayer. In this work, the influence of further processing of polyvinyl butyral film with non-uniform thickness is studied. Further processing may have a negative impact to the profile of PVB material.

Keywords: Technology, Automotive, Glass, Performance, PVB

\section{Introduction}

\subsection{Polyvinyl butyral principle}

Polyvinyl butyral as a material belonging to the group of polymers, concretely to the group called thermoplastic materials. Thermoplastic material is a material that is capable of softening or fusing when heated and of hardening again when cooled thermoplastic synthetic resins. Polyvinyl butyral (PVB) film as an interlayer for laminated automotive glass is used. The interlayer primarily fulfills a safety function in the windshield. Laminated glass with PVB interlayer is a type of safety glass that holds together when shattered. The windshield with polyvinyl butyral enabling the projection of information on the glass is the subject of this work. The PVB used for such purposes has a variable thickness profile that compensates the bent shape of the glass and allows image quality to be maintained by preventing double images. As the reflection of the light takes place on the outer and inner glass, a double image is created as standard. For this reason, the glass made for the Head Up Display has a certain angle, called the wedge angle, which allows the images to overlap so that no double image but only one image is observed. The processing effect of the synthesized and extruded polyvinyl butyral film is studied deeply [1].

\subsection{Polyvinyl butyral synthesis}

Polyvinyl butyral belongs into the group of polyvinyl acetals. These are formed by the condensation of polyvinyl alcohol with aldehydes in an acidic environment. They are produced either directly by hydrolysis of polyvinyl acetate in the presence of the correspon- ding aldehyde in a sulfuric or hydrochloric acid medium, or by acetylization of polyvinyl alcohol, again in the presence of a mineral acid [2].

The synthesis of polyvinyl butyral from acetic acid is a method used by PVB film suppliers of automotive plants. The chemical reaction takes place in 4 major steps. In the first step, acetylene and acetic acid react together in a sulfuric acid and mercuric sulfate environment, from which a vinyl acetate is formed by a chemical reaction.

$$
\underset{\text { Acetylene }}{\mathrm{HC} \equiv \mathrm{CH}}+\underset{\text { Acetic acid }}{\mathrm{CH}_{3} \mathrm{CO}_{2} \mathrm{H}} \underset{\mathrm{HgSO}_{4}}{\stackrel{\mathrm{H}_{2} \mathrm{SO}_{4}}{\longrightarrow}} \quad \mathrm{H}_{3} \mathrm{C}-\underset{\text { Vinyl acetate }}{\mathrm{C}}-\mathrm{O}-\underset{\mathrm{C}}{\mathrm{C}}=\mathrm{CH}_{2}
$$

Fig. 1 Synthesis of Vinyl acetate from Acetylene and Acetic Acid

Subsequently, the vinyl acetate is converted to polyvinyl acetate. At this stage, the monomer (vinyl acetate) becomes a polymer (polyvinyl acetate).

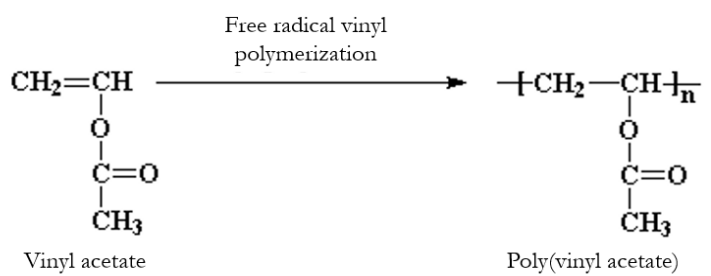

Fig. 2 Synthesis of poly(vinyl acetate) from vinyl acetate by free radical vinyl polymerization

In the second step, the polyvinyl acetate reacts to the polyvinyl alcohol (PVA) by hydrolytic cleavage in the presence of methanol with the residue formed by hydrolysis. 

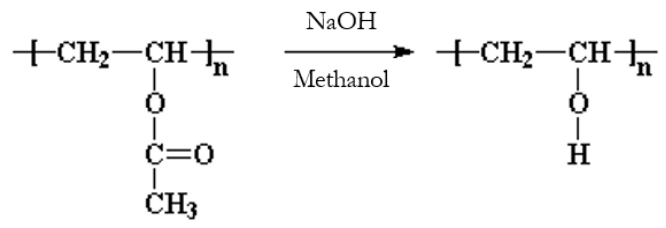

Poly(vinyl acetate)

Poly(vinyl alcohol) Fig. 3 Synthesis of poly(vinyl alcohol) from poly(vinyl acetate)
in methanol

In the third step, acetylization takes place by reacting the formed PVA with butanal to form polyvinyl butyral and hemiacetal (also hemiacetal). Adjacent molecules in the polymer chain react again to form acetal and water - this completes the acetylization and PVB synthesis as well [2-3].

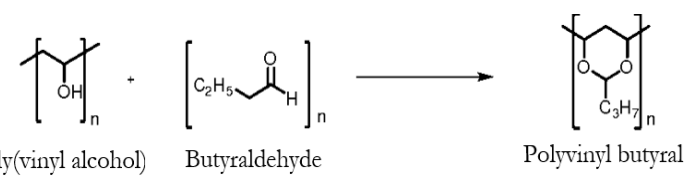

Fig. 4 Synthesis of polyvinyl butyral from polyvinyl alcohol and butyraldebyde

\subsection{Polyvinyl butyral film production}

PVB film is produced by extrusion of synthesized polyvinyl butyral - a white powder, which is mixed with plasticizer and additives. The use of triethylene glycol di-2-ethylhexanoate - abbreviated to 3G8 or $3 \mathrm{GEH}$ - as low-polarity plasticizer for polyvinyl butyral foils (PVB foil) has hitherto been restricted to PVB grades with a polyvinyl alcohol content below 19.5\% by weight [4].

The extrusion process is shown schematically in fig. 5. The polymer is fed into the press screw either in the solid state plastic extrusion or in the molten form by melt extrusion. When a solid substance is fed to the extruder, the extruder provides its basic extrusion function, but at this stage also the material must be comminuted and molten. In the latter case, it only serves as a piston exerting sufficient pressure to deliver the polymer to the mold. Another aspect for the division of the extrusion process is its fluidity - we distinguish two basic types, namely continuous extrusion and intermittent extrusion - dosing. PVB film is produced by plastic extrusion with a continuous extruder. The result is a continuous and fluid film production process that passes through a production process on one long endless belt on belt conveyors [5-7].

The first step (A) is to grind and melt the input material. After the material is molten, it is further blown under pressure into the extruder (B). The extruded material acquires a shape, according to the nozzle at the end of the extruder. After leaving the extruder, the input molten material becomes a PVB film. In order for the process to be complete, it is necessary for the film to pass between two embossing rollers
(C), which will extrude the required surface relief on the PVB film. The last step of production is zone (D). Here, by annealing in the area of viscous flow above glass transition temperature, we get rid of the stress in the material that arose during extrusion. Due to the effect of increased temperature during annealing, the humidity of the PVB film decreases, so the humidity is restored in the next step, which is to moisten the film.

Subsequently, the film leaves the production process and moves to a clean and refrigerated room of controlled temperature $(\mathrm{E})$, where it is wound into rolls of the required length and packed in aluminum containers that maintain the prescribed condition of the film.

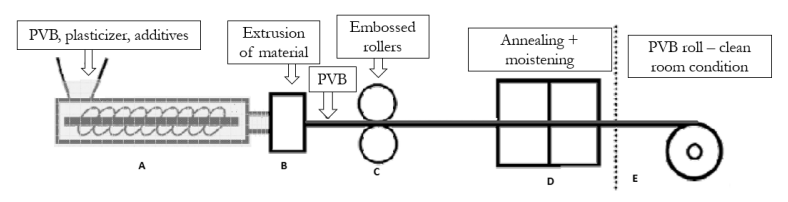

Fig. 5 Extrusion line for polyvinyl butyral

\subsection{Production of polyvinyl butyral film with non- uniform thickness profile (wedge PVB)}

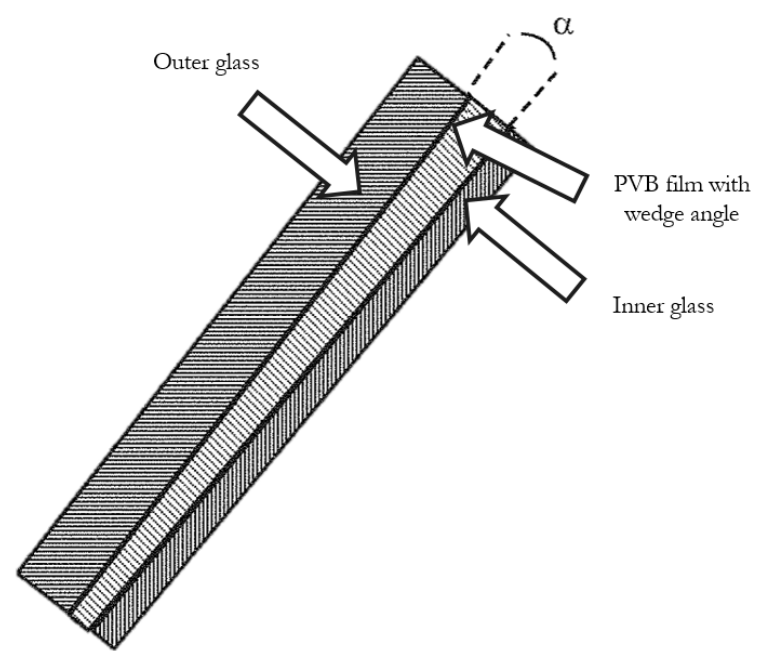

Fig. 6 Composition of windshield with a wedged PVB interlayer

The glass manufacturer has several options for securing the non-uniform thickness of PVB - calling the wedge angle in PVB - on the resulting windshield with a projection function - calling windshield with Head-Up Display. The basic principle to create a wedge angle in the PVB film is the material extrusion process, wherein a wedge-shaped extrusion die is used. The size of the wedge angle depends, among the other things, on the geometry of the concrete laminated windshield and the design arrangement of the head up display [8]. Further processing of the material with the wedge angle and its effect on the wedge angle value is deeply analysed in this work. The processing of wedge 
polyvinyl butyral film may have a negative impact on the thickness profile created in the PVB film by the extrusion process. The wedge PVB supplied by the manufacturer of the wedge rolls is unwound from the role in the first step. The PVB film is then heated up to shrinkage the tension and then cooled down in a controlled manner before being cut into wedge PVB sheets. Wedge PVB sheets are laminated with inner and outer glass sheets in the next step to manufacture the laminated windshields [9-10].

\subsection{Production of windshield using the PVB with non-uniform thickness for image projection}

The windshield with the wedge PVB interlayer is composed of the inner glass, wedge PVB and the outer glass. The glass is produced on the float line is extremely smooth, distortion-free glass used in many window applications. The glass floats on the tin and forms itself in the shape of the container. The float glass is a substance composed primarily of $\mathrm{Si}, \mathrm{Na}, \mathrm{Ca}$, $\mathrm{Mg}, \mathrm{Al}, \mathrm{K}, \mathrm{Fe}, \mathrm{Ti}, \mathrm{S}$ and $\mathrm{O}$ (commonly called "soda lime silicate" glass). $\mathrm{Pb}, \mathrm{Cr}, \mathrm{As}, \mathrm{Sb}, \mathrm{V}$ may be present in float glass as trace level contaminants, always below $20 \mathrm{ppm}$. Tinted glass may contain $\mathrm{Co}$, Se or $\mathrm{Ni}$, up to $200 \mathrm{ppm}$ [11]. The composition of float glass used for the windshield applications is a soda-silicate-based glass.

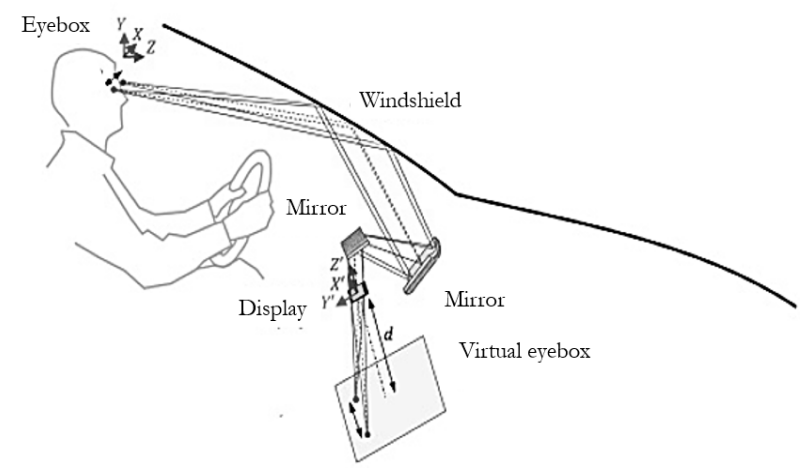

Fig. 7 Projection of images on the windshield containing on a windshield having a wedge PVB

The float glass is cut into sheets in required dimensions at the end of the float line. This glass sheets are transported into the plant that is able to bent glass sheets in the first step and laminate bent glass sheets with an interlayer in the second step. Windshield with Head-Up display function is manufactured by lamination of wedge PVB sheet with bent inner and outer glass sheets.

The Head-Up display function is provided by the projector integrated in the car body that projects images on the specific part of windshield. The HUD projection unit consists of a radiation source and a system of mirrors, both aspherical and flat mirror. To avoid any disturbing issues like horizontal or vertical double image, the wedged PVB interlayer is used to compensate the curviness of the windshield. The wedge angle of HUD PVB with lower thickness variation has been introduced by lately to reduce the projection errors to minimum [12]. A proper selection of the PVB wedge angle in the initial part of the development has a significant effect on the projected image quality. Improper wedge angle selection of PVB may lead to the errors in the projected image, like to the double image creation [10].

\subsection{Other production technologies of non-uniform thickness PVB film}

The other option is to create a wedge angle by stretching and shaping process by unwinding a flat, thermoplastic film after extrusion with uniform thickness from a supply roll and transporting same onwards to a heating drum. The next step is heating of the plastics film using a heating drum and transporting same onwards to compression rollers, pressing the plastics film in the nip between two heated compression rollers, wherein the nip has a clear width which corresponds to the cross-sectional profile of the plastics film to be produced, and transporting same onwards to a stretching apparatus. In the next step the PVB is stretched with a wedge-shaped cross-sectional profile in the stretching apparatus and transporting the plastics film onwards to a cooling apparatus. PVB film is cooled down in the cooling apparatus and PVB film is transported same onwards to a cutting apparatus. Than the PVB continuous film is cut into PVB sheets [13-14].

Another option how to manufacture the windshield with head-up display is the usage of coating on the glass. A laminated glass for implementing HUD function, the laminated glass comprising: an outer glass layer; an inner glass layer; a PVB film located between the outer glass layer and the inner glass layer; wherein the PVB film is of a uniform thickness, the laminated glass further comprises a reflection film [10].

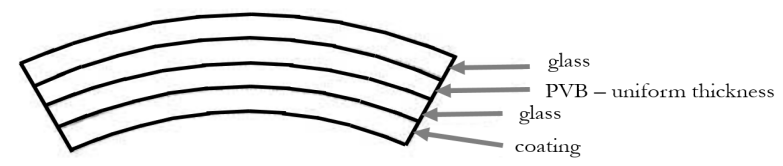

Fig. 8 Composition of the Head-Up display windshield using the reflection film

\subsection{Further processing steps of PVB film with non-uniform thickness}

This work is dedicated to the relaxation study of the wedge PVB sheets after cutting and its effect on the wedge angle value. The wedge angle should ideally remain the same as it exits the extrusion line, it means without any change of the wedge angle value in its further processing, but this is not always the case. Based on the selected cutting process, the wedge angle 
may vary, the process may typically change the value of the wedge angle based on the cutting conditions.

The consumer of wedge PVB film has to unwind the film from the roll and cut it to the PVB sheets. The PVB is normally heated up during the unwinding and cutting process due to the relaxation of remaining tension in PVB coming from the extrusion line. There is still some remaining tension in PVB after cutting to the sheets. Therefore the PVB sheets have to remain relaxing on the pallets after the cutting process and before lamination process. The relaxation time necessary to shrinkage the remaining tension and the recommended technology of relaxation is the objection of this work [13-14].

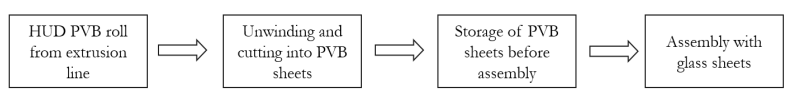

\section{Fig. 9 Process steps of HUD PVB material processing}

The process must be analyzed and understood properly to be able to correctly design the wedge angle of the wedge PVB roll on the extrusion line. One specific step of the technology is investigated deeply in this article. Relaxation of wedge PVB sheets before assembly has been analyzed deeply to better understand to the phenomena of wedge PVB relaxation [15].

\section{Experiment}

\subsection{Production of PVB sheets}

The cutting line is used to unwind the wedge PVB film from the roll after extrusion with a non-uniform thickness from the storage cylinder with the required wedge angle and transport it further to the heating drum. The next step is to heat the plastic foil using heating drums (two small and one large) up to $100^{\circ} \mathrm{C}$ and transport it further to another silver cylinder, where the temperature is up to $90^{\circ} \mathrm{C}$. In the next step, the PVB is stretched with a wedge-shaped cross-sectional profile in the stretching device and transports the plastic film further to the cooling device. The PVB film is cooled in a cooling device and the PVB film is transported further to the cutting device. The wedge PVB film is then cut into PVB sheets. The pallet traditionally stores from 20 to 80 wedge PVB sheets (based on the wedge angle), which relax for 72 hours. The bigger the wedge angle of PVB sheets we store on the pallet the lower amount of PVB sheets relaxes on the pallet. Wedge PVB sheets go to the next operation, which is glass lamination, after the relaxation is completed.

Relaxation of wedge PVB sheets before lamination can affect the wedge angle of the PVB sheets. The aim of this work is to evaluate the relaxation of PVB depending on the position of PVB on the pallet and to discuss the changes in wedge angle that occur during this operation. The aim is to choose a suitable relaxation time and to recommend the maximum number of pieces on the pallet for which similar relaxation conditions can be guaranteed so that the value of the wedge angle change is as similar as possible for the first and last wedge PVB sheets on the pallet.

\subsection{Relaxation study of wedge PVB sheets}

In the experiment, we measure the shrinkage of wedge PVB sheets from different parts of the pallet and we also measure the wedge angle of a given PVB sheet. We study the effect of PVB relaxation over time and its effect on the wedge of a given PVB. We measure the effect of the position of a given wedge PVB sheet on the pallet.

The relaxation time of 72 hours is analyzed and discused deeply in this article. Experimental ambient conditions are a clean room temperature of $18^{\circ} \mathrm{C}( \pm$ $\left.2{ }^{\circ} \mathrm{C}\right)$ with a relative humidity of $28 \%(+7 \%,-5 \%)$. Wedge PVB sheets have been measured in time based on their location on the pallet. The horizontal and vertical cross pattern in the dimension of $500 \mathrm{~mm}$ has been created on the PVB sheets. The dimensional changes in the cross pattern have been measured by calibrated meter and compared in time for differently localized PVB sheets on the pallet.

The wedge PVB sheet with a depicted cross pattern is in the fig. 10. Before the measurement, an axial cross is made on the PVB foil with the help of a black marker and a meter, while the length of the cross axes is $500 \mathrm{~mm}$ in the vertical direction - $y$-axis and $500 \mathrm{~mm}$ in the horizontal direction - $x$-axis. In the subsequent relaxation study, the changes in the cross pattern are analyzed in time (Fig. 10).

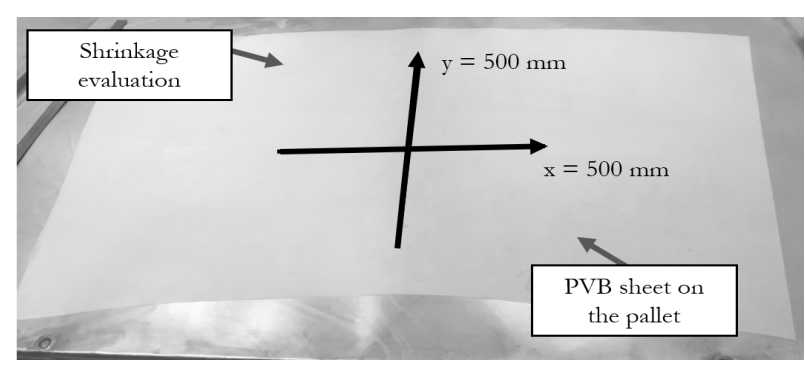

Fig. 10 Relaxation study of HUD PVB

\subsection{Evaluation of PVB sheets wedge angle}

The wedge angle, global or local, is evaluated by measuring the thickness of the HUD PVB (fig. 11). HUD PVB is measured with a contact table system for measuring the thickness of PVB film. The measurement principle is depicted in fig. 11. The sample for the measurement is the PVB material, which enters the mouth of the machine with the thinner side first. The two linear gauges rotate in opposite directions, allowing the PVB tape to move between the rollers and measure their entire distance. The range of measuring machines is $0.03-0.3 \mathrm{~mm}$, measuring length 10 
to $10000 \mathrm{~mm}$, measuring pitch $1 \mathrm{~mm}$, measuring force $0.6 \mathrm{~N}$.

\subsubsection{Evaluation of PVB global wedge angle}

Using the machine, we can evaluate the value of the global wedge angle of the PVB film. If we evaluate the global wedge angle of the film, it is a sample for measuring stripe PVB, which is cut from a PVB roll, or from a PVB sheet in the vertical direction. The global wedge angle is calculated for the HUD zone using the equation for the linear function $\mathrm{y}=m \mathrm{x}+\mathrm{b}$; where $m$ is the slope value.

\subsubsection{Evaluation of PVB local wedge angle varia- tion in the vertical direction}

If the local wedge angle of the PVB is evaluated, the sample is again a stripe PVB cut from the roll or from the PVB sheet in the vertical direction. The local wedge angle variation in the vertical direction is calculated for every $80 \mathrm{~mm}$ on the PVB on the basis of the same equation as described previously, using the equation for the linear function $\mathrm{y}=m \mathrm{x}+\mathrm{b}$; where $m$ is the slope value.

\subsubsection{Evaluation of PVB local wedge angle varia- tion in the horizontal direction}

When evaluating the local variation of the wedge angle in the horizontal direction, the PVB stripe is cut from the PVB roll or from the PVB sheet in the horizontal direction, regardless of which side the PVB stripe subsequently enters the machine mouth. The local wedge angle variation in the horisontal direction is calculated for every $80 \mathrm{~mm}$ on the PVB using the equation for the linear function $\mathrm{y}=m \mathrm{x}+\mathrm{b}$; where $m$ is the slope value.

PVB thickness measurement

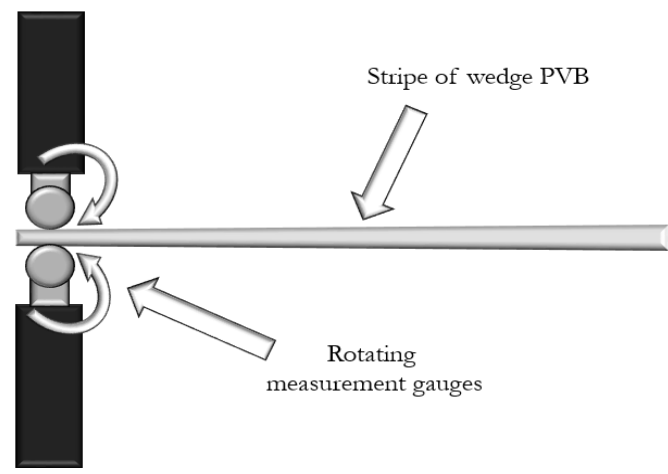

Fig. 11 Principle of the wedge PVB thickness measurement using 2 rotating measurement ganges that squeeze the PVB film

The aim of this work is to evaluate the effect of PVB relaxation on the wedge angle variation of PVB after cutting PVB from the roll. In the experiment, the wedge angle of the input roll in the vertical direction is first verified to confirm the wedge angle of the input material. A wedge PVB roll with a wedge of $0.33 \mathrm{mrad}$ with tolerances of $\pm 0.03 \mathrm{mrad}$ is used for the experiments.

\section{Measurement}

\subsection{Wedge angle measurement of the PVB roll}

The aim of this work is to evaluate the effect of PVB relaxation on the wedge angle of PVB after cutting PVB from the roll. A wedge PVB roll with a wedge of $0.33 \mathrm{mrad}$ with tolerances of $\pm 0.03 \mathrm{mrad}$ is used for the experiments. In the experiment, the wedge angle of the input roll in the vertical direction is first verified to confirm the correctness of the input material. The profile of the measured wedge PVB is in graph 1. The direction of the linear line is 0.3135 , which refers to the value of the global wedge angle $0.3135 \mathrm{mrad}$. The wedge angle value is within the tolerance of the input material.

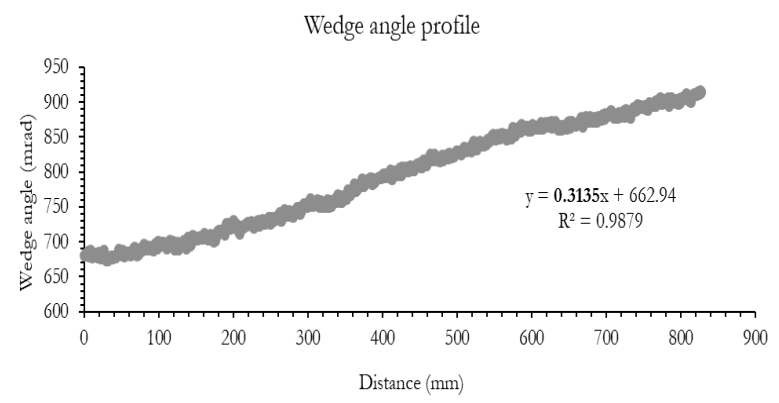

Graph 1 Wedge angle profile measured for wedge PVB

Subsequently, the wedge angle for 80 PVB sheets cut from the roll in the vertical direction is evaluated. The values are plotted in Graph 2. All measured values of the wedge angle of PVB sheets after cutting are in the specification of the input material (Graph 2-3, Table 1). With this evaluation, we verified that after cutting, all PVB sheets are still in the tolerances \pm 0.03 mrad.

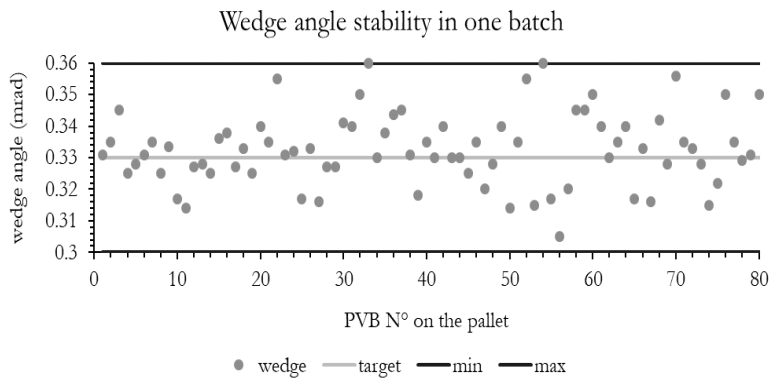

Graph 2 Wedge angle stability on wedge PVB roll 


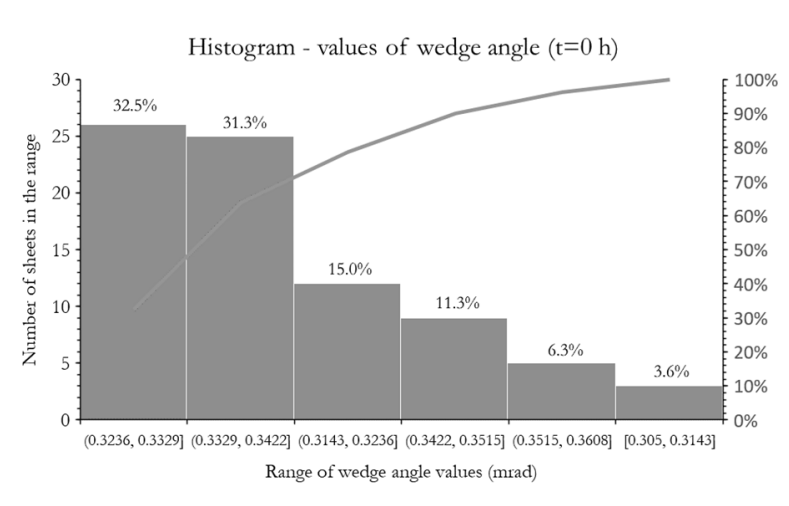

Graph 3 Histogram, number of PVB sheets after cutting (relaxation $t=0$ h) in the range of wedge angle values

Tab. 1 Calculated statistical parameters for 50 PVB stripes cut from the PVB roll

\begin{tabular}{|c|c|}
\hline $\mathrm{mrad}$ & parameter \\
\hline 0.3325888 & average \\
\hline 0.305 & $\min$ \\
\hline 0.36 & $\max$ \\
\hline 0.3315 & median \\
\hline 0.0114908 & standard deviation of the selection \\
\hline
\end{tabular}

The average calculated value of the wedge angle for 50 stripes of wedge PVB is $0.333 \mathrm{mrad}$. The minimal value is $0.305 \mathrm{~mm}$; the maximum values is $0.360 \mathrm{~mm}$; median value is $0.332 \mathrm{mrad}$, standard deviation is $0.011 \mathrm{mrad}$ (tab. 1). $32.5 \%$ of PVB sheets wedge angle values after cutting lay in the region between 0.3236 $\mathrm{mrad}$ and $0.3329 \mathrm{mrad}$. Another $31.3 \%$ of cut PVB sheets wedge angle values lay in the region between $0.3329 \mathrm{mrad}$ and $0.3422 \mathrm{mrad}$. Additional $15 \%$ of cut PVB sheets are in the range of $0.3143 \mathrm{mrad}$ to 0.3236 $\mathrm{mrad}$ concerning wedge angle after cutting. $11.3 \%$ of PVB sheets belongs to the region of $0.3422 \mathrm{mrad}$ to $0.3515 \mathrm{mrad}$. Only $6.3 \%$ of cut PVB sheets have the wedge angle value between $0.3515 \mathrm{mrad}$ and 0.3608 mrad. Remaining $3.6 \%$ of values are in the range of $0.305 \mathrm{mrad}$ to $0.3143 \mathrm{mrad}$ of the wedge angle value (see graph 3).

\subsection{Wedge angle and shrinkage measurement of cut PVB sheets}

After confirming the stability of the wedge angle of the input material, the stability of the wedge angle after processing the wedge PVB material on the cutting line is evaluated. The stability of the wedge angle is evaluated for different PVB sheets according to their position on the pallet (fig. 12). The relaxation study is performed as described in section 2.2. Wedge angle is evaluated for the wedge PVB sheets after cutting referring to the different position on the pallet.

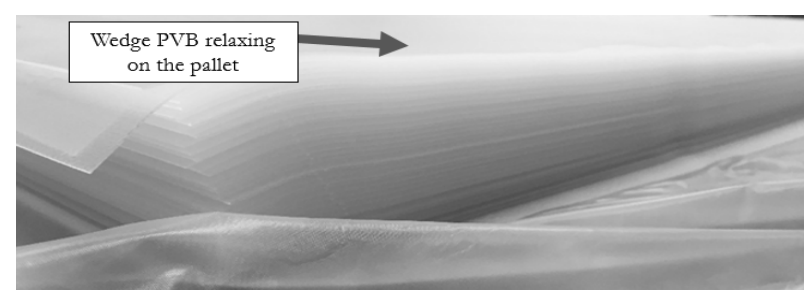

Fig. 12 Wedge PVB sheets relaxing on the pallet

Wedge angle is calculated in time for wedge PVB sheets located on the pallet in a different position. In the experiment, we decided to distinguish three basic positions on the pallet, namely the bottom position, the middle position and the upper position. To measure the PVB in the upper position, we refer to the behavior of the first five PVBs on the pallet (calculated from above). For the middle position of the PVB on the pallet, we refer to the 38th to the 42nd PVB on the pallet (calculated from above). And in the case of the bottom position on the pallet, we refer to the 76th to 80 th PVB on the pallet (counted from above).

\subsubsection{Wedge angle and shrinkage measurement of upper PVB sheets on the pallet}

The first measurement is done for upper 5 PVB sheets from the top on the pallet (tab. 2). We measure the change of the axis cross in time in the horizontal and in the vertical plane in time $0 \mathrm{~h}, 1 \mathrm{~h}, 2 \mathrm{~h}, 19 \mathrm{~h}, 24 \mathrm{~h}$, $48 \mathrm{~h}$ and $72 \mathrm{~h}$. We observe changes in the behavior of PVB film, the work is only mentioned as PVB shrinkage. PVB changes its dimensions in both the horizontal and vertical planes. With PVB shrinkage, we observe the behavior of PVB in terms of wedge angle. The correlation between PVB shrinkage in the vertical and horizontal direction and wedge angle changes is discussed below. Tab. 2 describes the measured values over time for a given 5 PVB sheets located in the upper position on the pallet. The table shows the values for horizontal and vertical shrinkage PVB in millimeters and the wedge angle values for the given sheets in mrad.

In graph 4. the behavior of the five upper PVB sheets in the palette in terms of vertical shrinkage is described. We can observe similar behavior for all 5 PVB sheets. PVB relaxation stabilizes after 48 hours.

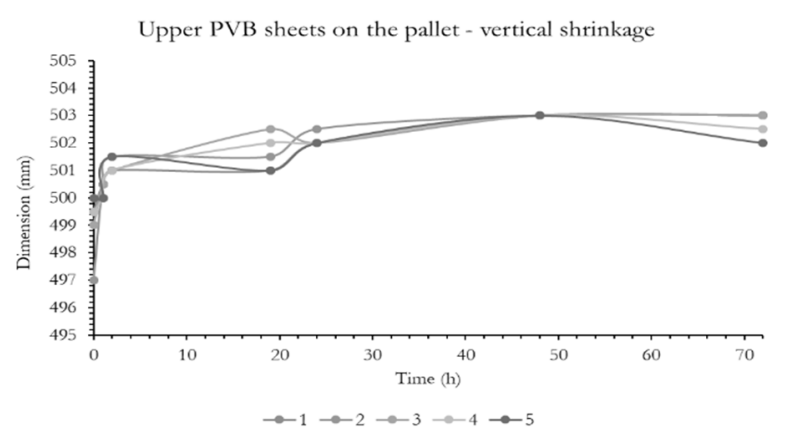

Graph 4 Evaluation of vertical shrinkage for 5 upper PVB sheets on the pallet in 72 hours 
Tab. 2 Evaluation of vertical shrinkage, horisontal shrinkage and wedge angle for upper 5 PVB sheets on the pallet in 72 hours

\begin{tabular}{|c|c|c|c|c|c|c|c|c|}
\hline \multicolumn{9}{|c|}{ Upper PVB sheet on the pallet } \\
\hline time $(\mathrm{h})$ & PVB sheet & 0 & 1 & 2 & 19 & 24 & 48 & 72 \\
\hline \multirow{6}{*}{ vertically } & 1 & 499.5 & 500.5 & 501.0 & 501.0 & 502.0 & 503.0 & 503.0 \\
\hline & 2 & 497.0 & 500.5 & 501.5 & 501.5 & 502.5 & 503.0 & 503.0 \\
\hline & 3 & 499.0 & 500.5 & 501.0 & 502.5 & 502.0 & 503.0 & 503.0 \\
\hline & 4 & 499.5 & 500.0 & 501.0 & 502.0 & 502.0 & 503.0 & 502.5 \\
\hline & 5 & 500.0 & 500.0 & 501.5 & 501.0 & 502.0 & 503.0 & 502.0 \\
\hline & Average & 499.0 & 500.3 & 501.2 & 501.6 & 502.1 & 503.0 & 502.7 \\
\hline \multirow{6}{*}{ horisontally } & 1 & 499.5 & 498.5 & 498.0 & 497.5 & 497.5 & 496.5 & 496.5 \\
\hline & 2 & 498.0 & 497.0 & 496.5 & 496.0 & 496.0 & 495.0 & 495.0 \\
\hline & 3 & 501.0 & 500.0 & 495.5 & 495.0 & 494.0 & 495.0 & 495.5 \\
\hline & 4 & 500.0 & 499.5 & 499.5 & 497.0 & 496.5 & 495.0 & 495.0 \\
\hline & 5 & 499.5 & 498.5 & 498.0 & 497.0 & 496.0 & 495.5 & 495.0 \\
\hline & Average & 499.6 & 498.7 & 497.5 & 496.5 & 496.0 & 495.4 & 495.4 \\
\hline \multirow{6}{*}{ wedge angle (mrad) } & 1 & 0.331 & 0.335 & 0.322 & 0.319 & 0.315 & 0.310 & 0.310 \\
\hline & 2 & 0.350 & 0.345 & 0.335 & 0.320 & 0.295 & 0.300 & 0.300 \\
\hline & 3 & 0.345 & 0.345 & 0.330 & 0.325 & 0.320 & 0.315 & 0.315 \\
\hline & 4 & 0.341 & 0.335 & 0.330 & 0.330 & 0.325 & 0.300 & 0.300 \\
\hline & 5 & 0.350 & 0.335 & 0.330 & 0.325 & 0.330 & 0.320 & 0.325 \\
\hline & average & 0.343 & 0.339 & 0.329 & 0.324 & 0.317 & 0.309 & 0.310 \\
\hline
\end{tabular}

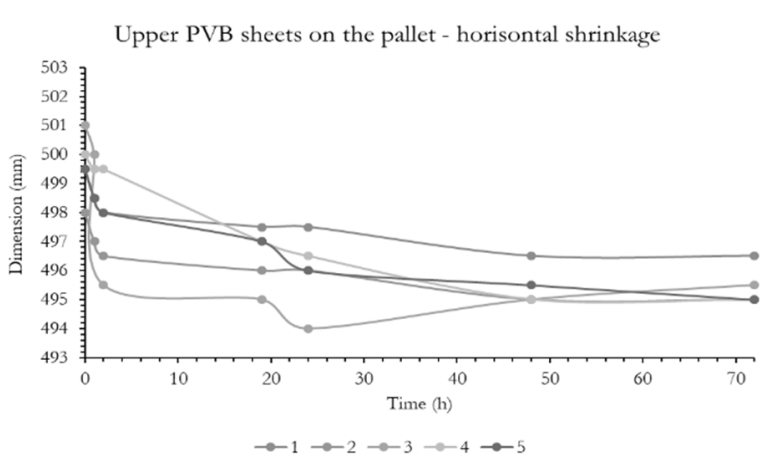

Graph 5 Evaluation of horisontal shrinkage for 5 upper $P V B$ sheets on the pallet in 72 hours

In graph 5. the behavior of the five upper PVB sheets on the pallet in terms of horizontal shrinkage is described. We can observe similar behavior for all 5 PVB sheets. PVB relaxation stabilizes after 48 hours.

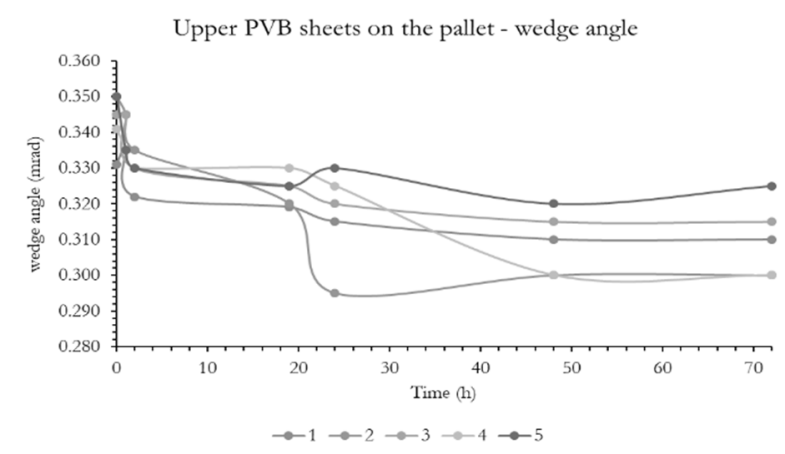

Graph 6 Evaluation of wedge angle for 5 upper PVB sheets on the pallet in 72 hours
In graph 6. the behavior of the five upper PVB sheets in the palette in terms of the wedge angle value over time is described. We can observe similar behavior for all 5 PVB sheets. The wedge angle value changes and stabilizes after 48 hours.

The top 5 PVB sheets from above in the palette relax in time and change vertically in average from by $3.7 \mathrm{~mm}$ from $499.0 \mathrm{~mm}$ to $502.7 \mathrm{~mm}$, horizontally in average by $4.6 \mathrm{~mm}$ from $499.6 \mathrm{~mm}$ to $495.0 \mathrm{~mm}$. PVB shrinkage occurs mainly in the first two hours after cutting the PVB wedge onto the pallet. Relaxation stops after 48 hours. The wedge angle changes over time, with the most significant change in the wedge angle occurring in the first two hours after cutting the PVB onto the pallet. The wedge angle value changes in average for 5 wedge sheets by $0.033 \mathrm{mrad}$ from $0.343 \mathrm{mrad}$ to $0.310 \mathrm{mrad}$ (tab. 2, Graph 4, 5, 6).

\subsubsection{Wedge angle and shrinkage measurement of middle PVB sheets on the pallet}

The second measurement is performed for the middle 5 PVB sheets from above on the pallet (tab. 3). We measure the change of the axis cross in time in the horizontal and in the vertical plane in time $0 \mathrm{~h}, 1 \mathrm{~h}, 2 \mathrm{~h}$, 19h, 24h, 48h and 72h. We observe a shrinkage of PVB. PVB changes its dimensions in the horizontal and vertical planes. For PVB shrinkage, we monitor the behavior of PVB in terms of wedge angle. The correlation between PVB shrinkage in the vertical and horizontal directions and wedge angle changes is discussed below. Tab. 3 describes the measured values over time for a given 5 PVB sheets placed in the middle position on the pallet. The tab. 3 shows the values 
of horizontal and vertical shrinkage of PVB in millimeters and the values of the wedge angle for the given sheets in mrad.

Tab. 3 Evaluation of vertical shrinkage, horisontal shrinkage and wedge angle for 5 PVB sheets located in the middle part of the pallet in 72 bours

\begin{tabular}{|c|c|c|c|c|c|c|c|c|}
\hline \multicolumn{9}{|c|}{ Middle PVB sheets on the pallet } \\
\hline time $(\mathrm{h})$ & & 0 & 1 & 2 & 19 & 24 & 48 & 72 \\
\hline \multirow{6}{*}{ vertically } & 38 & 501.0 & 501.0 & 501.5 & 502.0 & 502.0 & 502.0 & 502.0 \\
\hline & 39 & 500.0 & 500.5 & 501.0 & 501.5 & 502.5 & 503.5 & 503.0 \\
\hline & 40 & 501.0 & 501.5 & 501.5 & 501.0 & 502.0 & 502.0 & 502.0 \\
\hline & 41 & 499.0 & 502.5 & 502.0 & 501.5 & 502.0 & 502.5 & 502.5 \\
\hline & 42 & 499.0 & 500.0 & 501.0 & 502.0 & 502.0 & 503.0 & 503.0 \\
\hline & Average & 500.0 & 501.1 & 501.4 & 501.6 & 502.1 & 502.6 & 502.5 \\
\hline \multirow{6}{*}{ horisontally } & 38 & 499.5 & 499.5 & 499.0 & 498.5 & 498.0 & 497.5 & 497.5 \\
\hline & 39 & 499.0 & 498.0 & 497.0 & 497.0 & 497.0 & 497.0 & 496.5 \\
\hline & 40 & 499.0 & 499.0 & 498.5 & 498.5 & 498.0 & 497.5 & 497.0 \\
\hline & 41 & 500.0 & 499.5 & 497.5 & 497.0 & 497.5 & 496.0 & 496.0 \\
\hline & 42 & 501.0 & 499.0 & 498.0 & 497.5 & 496.5 & 495.5 & 495.5 \\
\hline & Average & 499.7 & 499.0 & 498.0 & 497.7 & 497.4 & 496.7 & 496.5 \\
\hline \multirow{6}{*}{ wedge angle (mrad) } & 38 & 0.340 & 0.335 & 0.335 & 0.330 & 0.325 & 0.320 & 0.320 \\
\hline & 39 & 0.350 & 0.330 & 0.325 & 0.325 & 0.315 & 0.315 & 0.315 \\
\hline & 40 & 0.360 & 0.350 & 0.340 & 0.335 & 0.315 & 0.305 & 0.310 \\
\hline & 41 & 0.330 & 0.325 & 0.320 & 0.315 & 0.315 & 0.310 & 0.310 \\
\hline & 42 & 0.338 & 0.341 & 0.334 & 0.320 & 0.315 & 0.315 & 0.315 \\
\hline & average & 0.344 & 0.336 & 0.331 & 0.325 & 0.317 & 0.313 & 0.314 \\
\hline
\end{tabular}

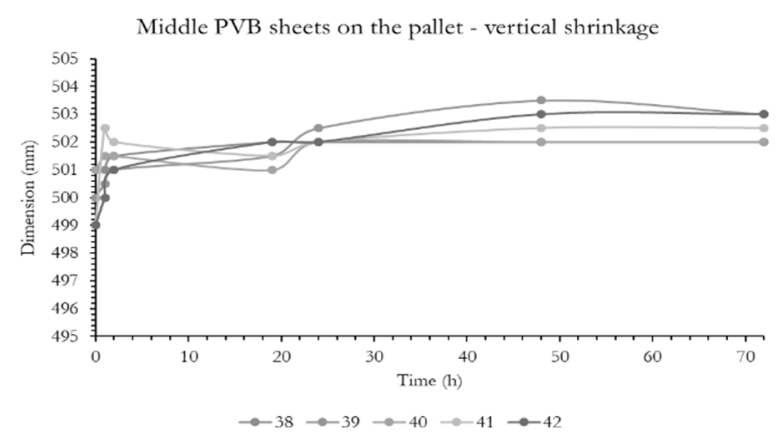

Graph 7 Evaluation of vertical shrinkage for 5 middle PVB sheets on the pallet in 72 hours

In graph 7. the behavior of the five middle PVB sheets on the pallet in terms of vertical shrinkage is described. We can observe similar behavior for all 5 PVB sheets. PVB relaxation stabilizes after 48 hours.

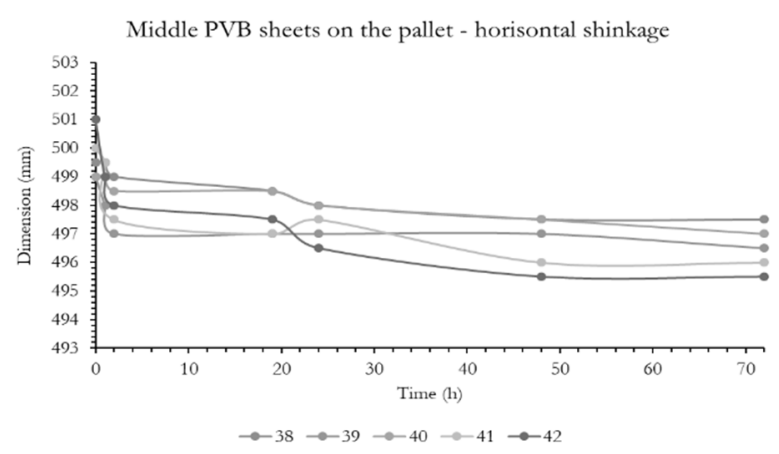

Graph 8 Evaluation of horisontal shrinkage for 5 middle PVB sheets on the pallet in 72 hours
In graph 8 . the behavior of the five middle PVB sheets on the pallet in terms of horizontal shrinkage is described. We can observe similar behavior for all 5 PVB sheets. PVB relaxation stabilizes after 48 hours.

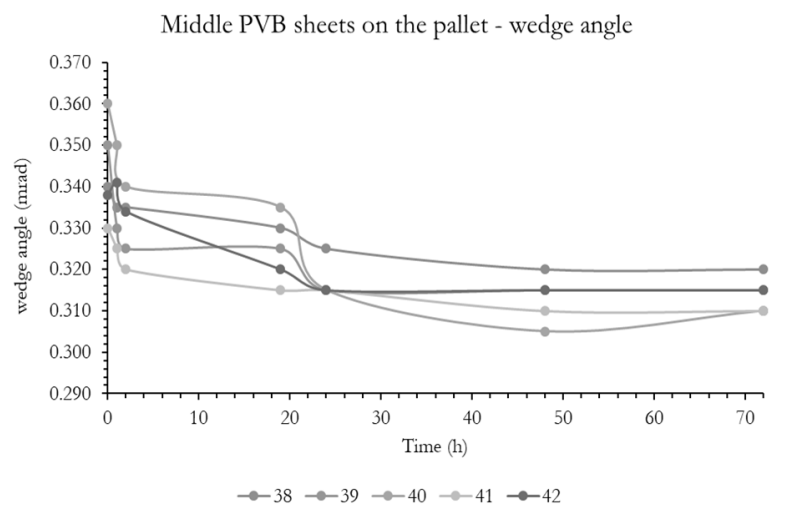

Graph 9 Evaluation of wedge angle for middle $5 \mathrm{PVB}$ sheets on the pallet in 72 hours

In graph 9. the behavior of the five middle PVB sheets on the pallet in terms of the wedge angle value over time is described. We can observe similar behavior for all 5 PVB sheets. The wedge angle value changes and stabilizes after 48 hours.

The middle 5 PVB sheets on the pallet relax in time and change vertically in average from by $2.5 \mathrm{~mm}$ from $500.0 \mathrm{~mm}$ to $502.5 \mathrm{~mm}$, horizontally in average from by $3.2 \mathrm{~mm}$ from $499.7 \mathrm{~mm}$ to $496.5 \mathrm{~mm}$. PVB shrinkage occurs mainly in the first two hours after cutting 
the PVB wedge onto the pallet. Relaxation stops after 48 hours. The wedge angle changes over time, with the most significant change in the wedge angle occurring in the first two hours after cutting the PVB onto the pallet. The wedge angle value changes in average for 5 wedge sheets by $0.031 \mathrm{mrad}$ from $0.345 \mathrm{mrad}$ to 0.314 mrad (tab. 3, Graph 7, 8, 9).

\subsubsection{Wedge angle and shrinkage measurement of middle PVB sheets on the pallet}

The third measurement is performed for the bottom 5 PVB sheets from above on the pallet (tab. 4). We measure the change of the axis cross in time in the horizontal and in the vertical plane in time $0 \mathrm{~h}, 1 \mathrm{~h}, 2 \mathrm{~h}$, $19 \mathrm{~h}, 24 \mathrm{~h}, 48 \mathrm{~h}$ and $72 \mathrm{~h}$. We observe a shrinkage of PVB. PVB slightly changes its dimensions in the horizontal and vertical planes. For PVB shrinkage, we monitor the behavior of PVB in terms of wedge angle. The correlation between PVB shrinkage in the vertical and horizontal directions and wedge angle changes is discussed below. Tab. 4 describes the measured values over time for a given 5 PVB sheets placed in the bottom position on the pallet. The tab. 4 shows the values of horizontal and vertical shrinkage of PVB in millimeters and the values of the wedge angle for the given sheets in mrad.

Tab. 4 Evaluation of vertical shrinkage, horisontal shrinkage and wedge angle for 5 PVB sheets located at the bottom part of the pallet in 72 bours

\begin{tabular}{|c|c|c|c|c|c|c|c|c|}
\hline \multicolumn{9}{|c|}{ Bottom PVB sheets on the pallet } \\
\hline time $(\mathrm{h})$ & & 0 & 1 & 2 & 19 & 24 & 48 & 72 \\
\hline \multirow{6}{*}{ vertically } & 76 & 500.5 & 500.5 & 500.0 & 500.0 & 500.5 & 500.0 & 500.0 \\
\hline & 77 & 500.0 & 500.0 & 500.5 & 499.5 & 499.5 & 500.0 & 499.5 \\
\hline & 78 & 501.0 & 500.5 & 500.0 & 501.0 & 500.5 & 500.0 & 499.0 \\
\hline & 79 & 500.0 & 500.5 & 500.0 & 499.5 & 499.5 & 499.5 & 500.0 \\
\hline & 80 & 499.5 & 499.0 & 498.5 & 499.0 & 498.5 & 498.0 & 498.0 \\
\hline & Average & 500.2 & 500.1 & 499.8 & 499.8 & 499.7 & 499.5 & 499.3 \\
\hline \multirow{6}{*}{ horisontally } & 76 & 499.5 & 499.5 & 499.5 & 498.5 & 499.0 & 498.5 & 498.5 \\
\hline & 77 & 499.0 & 498.5 & 498.5 & 498.0 & 498.0 & 498.0 & 498.0 \\
\hline & 78 & 500.0 & 501.0 & 500.0 & 499.0 & 499.5 & 499.0 & 499.0 \\
\hline & 79 & 500.0 & 499.5 & 499.5 & 499.5 & 499.5 & 499.5 & 499.0 \\
\hline & 80 & 499.5 & 499.0 & 499.0 & 498.5 & 498.0 & 498.0 & 498.5 \\
\hline & Average & 499.6 & 499.5 & 499.3 & 498.7 & 498.8 & 498.6 & 498.6 \\
\hline \multirow{6}{*}{ wedge angle (mrad) } & 76 & 0.345 & 0.339 & 0.333 & 0.335 & 0.335 & 0.329 & 0.329 \\
\hline & 77 & 0.350 & 0.350 & 0.345 & 0.335 & 0.340 & 0.330 & 0.330 \\
\hline & 78 & 0.340 & 0.345 & 0.330 & 0.335 & 0.330 & 0.325 & 0.325 \\
\hline & 79 & 0.330 & 0.330 & 0.335 & 0.315 & 0.320 & 0.320 & 0.320 \\
\hline & 80 & 0.335 & 0.335 & 0.330 & 0.330 & 0.330 & 0.325 & 0.325 \\
\hline & average & 0.340 & 0.340 & 0.335 & 0.330 & 0.331 & 0.326 & 0.326 \\
\hline
\end{tabular}

In graph 10. the behavior of the five bottom PVB sheets on the pallet in terms of vertical shrinkage is described. We can observe similar behavior for all 5 PVB sheets. PVB relaxation stabilizes after 48 hours.

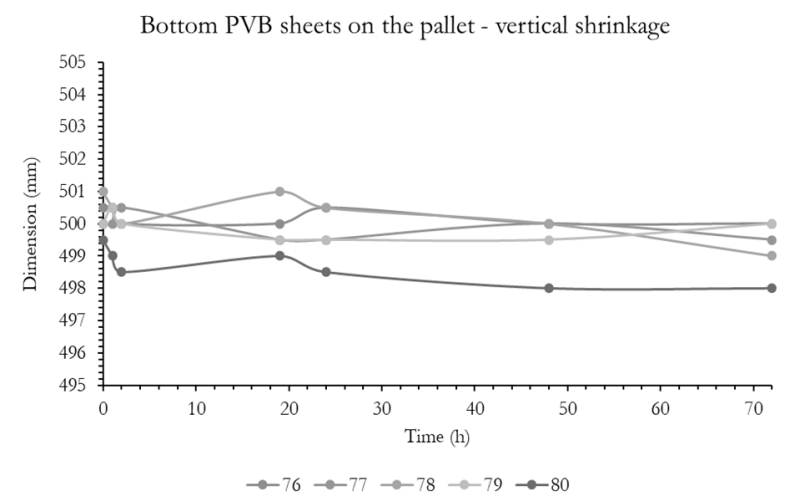

Graph 10 Evaluation of bottom 5 PVB sheets on the pallet for vertical shrinkage
In graph 11. the behavior of the five bottom PVB sheets on the pallet in terms of horizontal shrinkage is described. We can observe similar behavior for all 5 PVB sheets. PVB relaxation stabilizes after 48 hours.

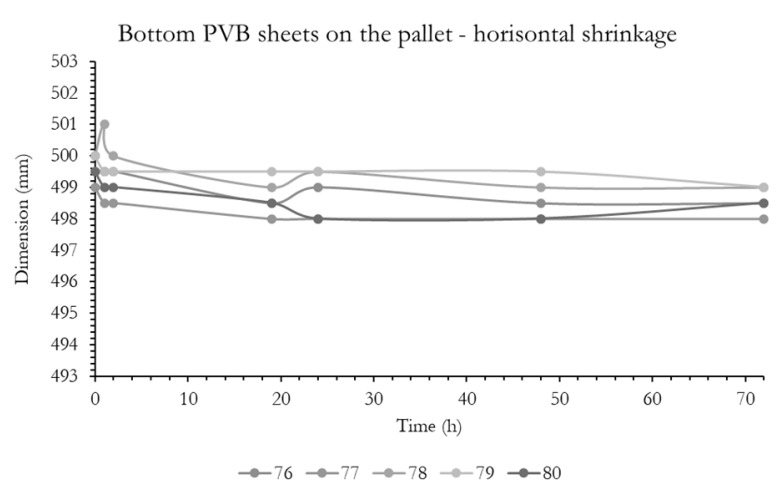

Graph 11 Evaluation of bottom 5 PVB sheets on the pallet for horizontal shrinkage 
In graph 12. the behavior of the five bottom PVB sheets on the pallet in terms of the wedge angle value over time is described. We can observe similar behavior for all 5 PVB sheets. The wedge angle value changes and stabilizes after 48 hours.

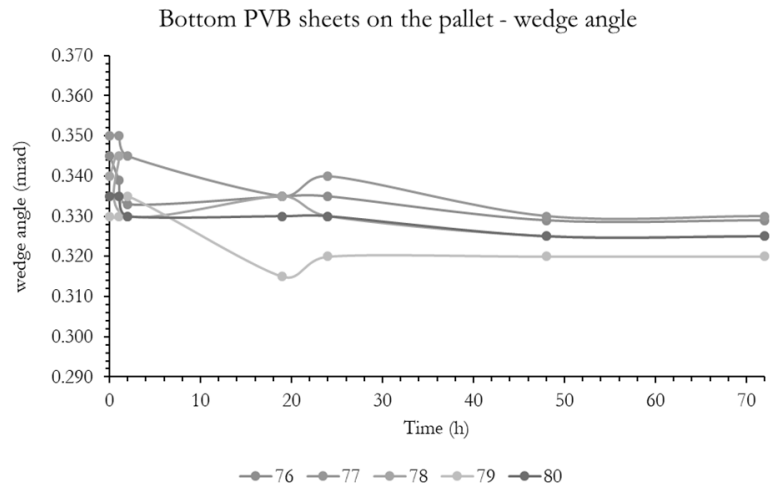

Graph 12 Evaluation of PVB wedge angle of bottom 5 $P V B$ sheets on the pallet

The bottom 5 PVB sheets on the pallet relax slightly in time. We don't observe any significant shrinkage in the vertical direction, we observe the change in width in average by $0.9 \mathrm{~mm}$ from 500.2 to $499.3 \mathrm{~mm}$. We observe slight shrinkage in the horisontal direction by $1 \mathrm{~mm}$. The wedge angle is changing by $0.014 \mathrm{mrad}$ from $0.34 \mathrm{mrad}$ to $0.326 \mathrm{mrad}$. PVB shrinkage occurs negligibly during 72 hours after cutting the PVB wedge onto the pallet. Relaxation stops after 48 hours. The wedge angle slightly changes over time. The wedge angle value changes in average for 5 wedge sheets by $0.014 \mathrm{mrad}$ from $0.340 \mathrm{mrad}$ to $0.326 \mathrm{mrad}$ (tab. 4, Graph 10, 11, 12).

\subsubsection{Evaluation of the wedge angle value based on the position of PVB sheets on the pallet}

The graph 13 depicts the correlation among the wedge angle value for a dedicated PVB sheet on the pallet and its position on the pallet.

We observe the change in the wedge angle value for all measured PVB sheets. The intensity of the wedge angle change depends on the position of the PVB on the pallet (graph 13). The most significant change in the wedge angle value is detected for the upper 5 PVB sheets on the pallet (PVB sheets 1-5). Less significant change is observable for the middle PVB sheets on the pallet (PVB sheets 38-42). The lowest change is detected for 5 bottom PVB sheets on the pallet (PVB sheets 76-80).

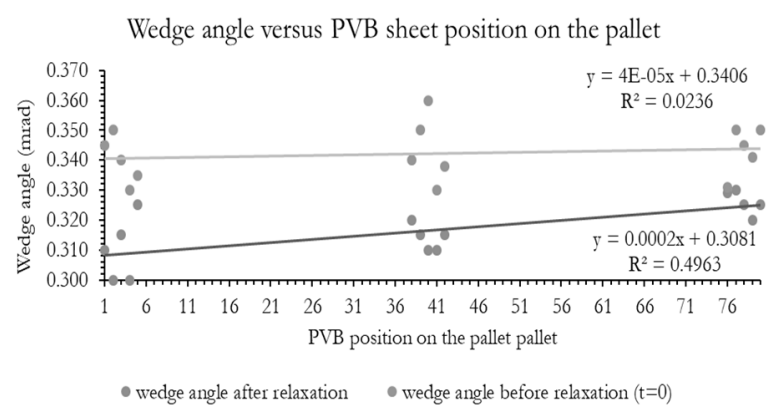

Graph 13 Wedge angle of PVB sheets before and after relaxation

\subsubsection{Evaluation of vertical shrinkage based on the position of PVB sheets on the pallet}

Vertical shrinkage is compared for each position of the wedge PVB on the pallet to see the correlation among vertical shrinkage and position of the wedge PVB on the pallet. Vertical shrinkage is almost completely stopped after 48 hours. Upper wedge PVB sheets shrink by $0.86 \%$ in 72 hours; middle wedge PVB sheets shrink by $0.54 \%$ in 72 hours and bottom wedge PVB sheets shrink by $0.18 \%$ in 72 hours. The maximal change in vertical shrinkage is observed for upper PVB sheets on the pallet by $0.86 \%$ in 72 hours. Wedge PVB shrinks during last 24 hours by $0.06 \%$ from $0.80 \%$ to $0.86 \%$ for the upper PVB sheets on the pallet. There is almost no shrinkage observable during last 24 hours for PVB sheets in the middle part of pallet (change by $0.02 \%$ from $0.52 \%$ to $0.54 \%$ ). There is no change in the vertical shrinkage for wedge PVB sheets located at the bottom part of the pallet during last 24 hours (tab. 5).

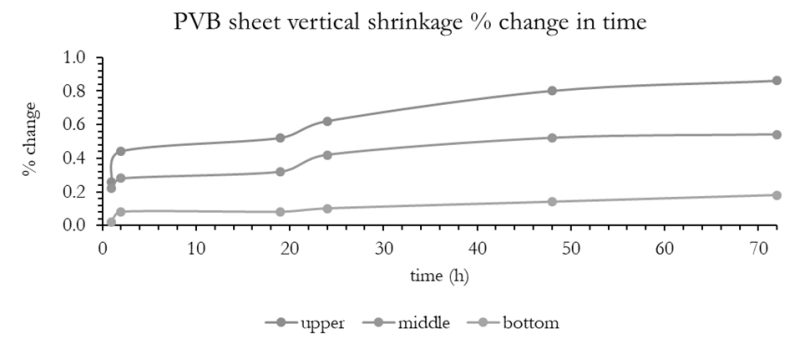

Graph 14 Vertical shrinkage of wedge PVB sheets based on their position on the pallet

Tab. 5 Vertical shrinkage of PVB sheets in 72 hours based on their position on the pallet

\begin{tabular}{|c|c|c|c|c|c|c|c|c|}
\hline \multicolumn{7}{|c|}{ vertical shrinkage of PVB sheets \% change in time } \\
\hline time $(\mathrm{h})$ & 0 & 1 & 2 & 19 & 24 & 48 & 72 & Suma \% \\
\hline top & 0.00 & 0.26 & 0.44 & 0.52 & 0.62 & 0.80 & 0.86 & 0.86 \\
\hline middle & 0.00 & 0.22 & 0.28 & 0.32 & 0.42 & 0.52 & 0.54 & 0.54 \\
\hline bottom & 0.00 & 0.02 & 0.08 & 0.08 & 0.10 & 0.14 & 0.18 & 0.18 \\
\hline
\end{tabular}




\subsubsection{Evaluation of horisontal shrinkage based on the position of PVB sheets on the pallet}

Horisontal shrinkage is compared for each position of the wedge PVB on the pallet to see the correlation among horisontal shrinkage and position of the wedge PVB on the pallet. Upper wedge PVB sheets shrink by $0.84 \%$ in 72 hours; middle wedge PVB sheets shrink by $0.64 \%$ in 72 hours and bottom wedge PVB shrink by $0.24 \%$ in 72 hours. The maximal change in horisontal shrinkage is observed for upper PVB sheets on the pallet by $0.84 \%$ in 72 hours. Horisontal shrinkage is stopped after 48 hours for upper, middle and bottom wedge PVB sheets on the pallet (tab. 6).

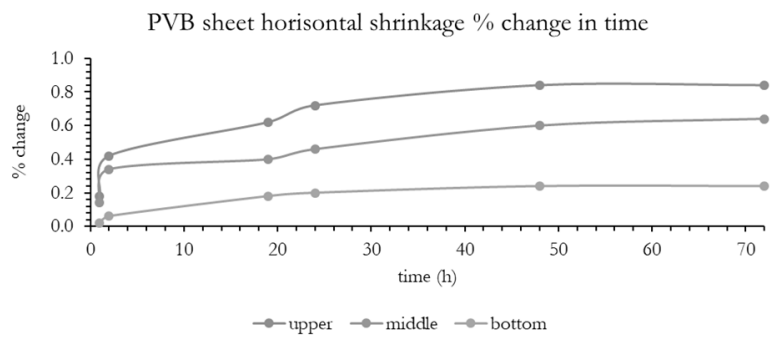

Graph 15 Horisontal shrinkage of PVB sheets based on their position on the pallet

Tab. 6 Horisontal shrinkage of PVB sheets in 72 hours based on their position on the pallet

\begin{tabular}{|c|c|c|c|c|c|c|c|c|}
\hline \multicolumn{7}{|c|}{ horisontal shrinkage of PVB sheets \% change in time } \\
\hline time $(\mathrm{h})$ & 0 & 1 & 2 & 19 & 24 & 48 & 72 & Suma \% \\
\hline top & 0.00 & 0.18 & 0.42 & 0.62 & 0.72 & 0.84 & 0.84 & 0.84 \\
\hline middle & 0.00 & 0.14 & 0.34 & 0.40 & 0.46 & 0.60 & 0.64 & 0.64 \\
\hline bottom & 0.00 & 0.02 & 0.06 & 0.18 & 0.20 & 0.24 & 0.24 & 0.24 \\
\hline
\end{tabular}

\subsubsection{Evaluation of wedge angle change based on the position of PVB sheets on the pallet}

Wedge angle change is compared for each position of the wedge PVB on the pallet to see the correlation among the change of the wedge angle and position of the wedge PVB on the pallet. Wedge angle change is stopped within 48 hours. There is no wedge angle change during last 24 hours of PVB sheets relaxation. The maximal change in the wedge angle value is observed for upper PVB sheets on the pallet by $10.31 \%$ in 72 hours. The wedge angle changes by $9.46 \%$ for middle PVB sheets on the pallet and by $4.76 \%$ for the bottom PVB sheets on the pallet (tab. 7).

Tab. 7 PVB wedge angle change in 72 hours based on the position of PVB sheets on the pallet

\begin{tabular}{|c|c|c|c|c|c|c|c|c|}
\hline \multicolumn{8}{|c|}{ wedge angle of PVB sheets \% change in time } \\
\hline time $(\mathrm{h})$ & 0 & 1 & 2 & 19 & 24 & 48 & 72 & Suma \% \\
\hline top & 0.00 & 1.28 & 4.08 & 5.71 & 7.69 & 10.02 & 10.31 & 10.31 \\
\hline middle & 0.00 & 2.44 & 4.01 & 5.69 & 8.01 & 9.17 & 9.46 & 9.46 \\
\hline bottom & 0.00 & 0.06 & 1.59 & 2.94 & 3.23 & 4.76 & 4.76 & 4.76 \\
\hline
\end{tabular}

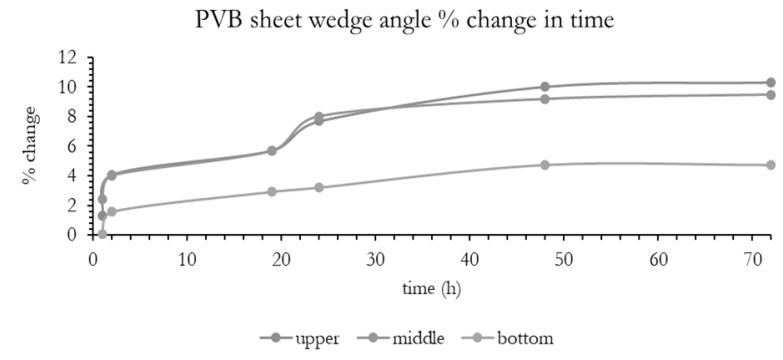

Graph 16 Change of wedge angle of PVB sheets based on their position on the pallet

\subsubsection{Correlation between horisontal and vertical shrinkage and wedge angle of PVB sheets}

The correlation among vertical shrinkage, horisontal shrinkage and wedge angle change is analysed more deeply. We can observe linear correlation among ver- tical shrinkage and horisontal shrinkage for all evaluated wedge PVB sheets.

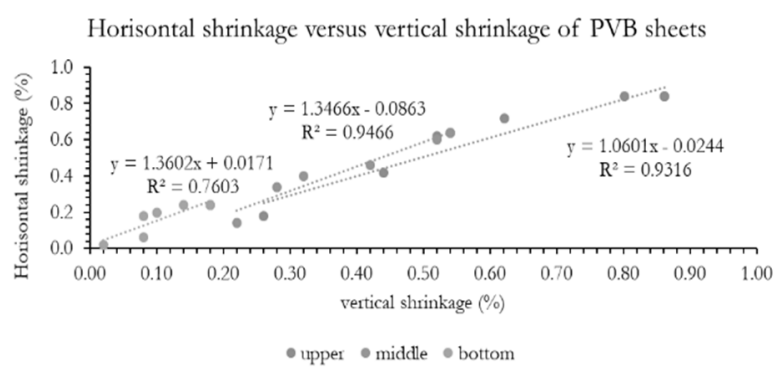

Graph 17 Correlation among horisontal shrinkage and vertical shrinkage of PVB sheets

We can observe the linear correlation between horisontal shrinkage and wedge angle value for all evaluated wedge PVB sheets. 
Horisontal shrinkage versus wedge angle of PVB sheets

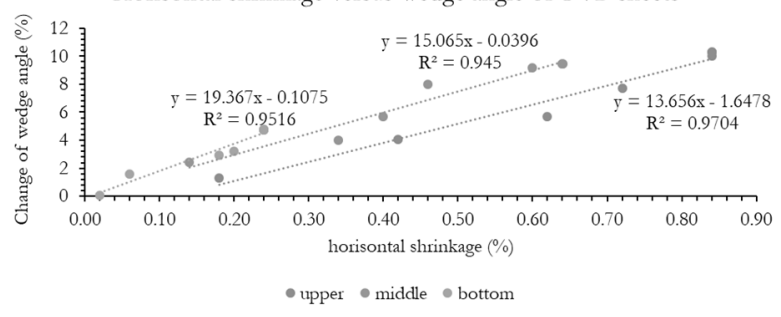

Graph 18 Correlation among horisontal shrinkage and wedge angle value of $P V B$ sheets

We can observe the linear correlation between vertical shrinkage and wedge angle value for all evaluated wedge PVB sheets.

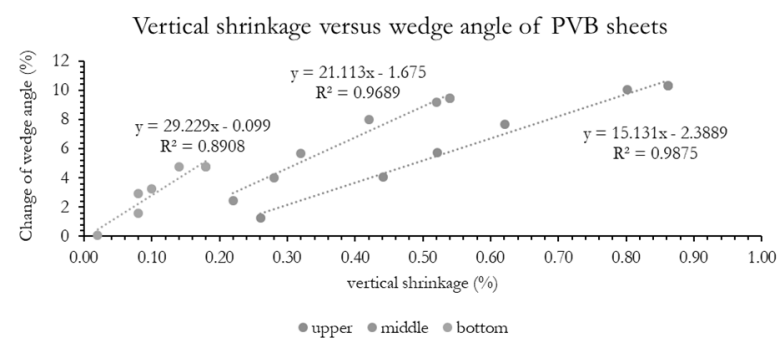

Graph 19 Correlation among vertical shrinkage and wedge angle value of $P V B$ sheets.

\section{Discussion and results}

The value of the PVB wedge angle can be slightly changed by the selected cutting process. The wedge angle calculated for a wedge PVB cylinder with a specification of $0.33 \pm 0.03 \mathrm{mrad}$ is $0.3135 \mathrm{mrad}$ (graph $1)$, ie within tolerances. The average value of the wedge angle calculated after cutting the wedge PVB foil into wedge PVB sheets is $0.343 \mathrm{mrad}$ for the upper wedge PVB sheets (tab. 2) on the pallet; 0.344 for medium wedge PVB sheets (tab. 3) on the pallet and $0.340 \mathrm{mrad}$ for wedge PVB sheets at the bottom of the pallet (tab. 4). The wedge angle increases by 0.0275 mrad for the upper PVB sheets on the pallet during the cutting process, by $0.0305 \mathrm{mrad}$ for the middle PVB sheets on the pallet and by $0.0265 \mathrm{mrad}$ for the bottom PVB sheets on the pallet. When selecting a suitable PVB wedge, the change in the wedge angle value caused by the selected PVB cutting process must be taken into account.

The shinkage of the wedge PVB sheets after the cutting process again affects the value of the wedge angle, but in this case the value of the wedge decreases. The change in the wedge angle is easily observable with PVB sheets, which are placed at the top of the pallet (PVB sheets 1-5) (graph 6). The lowest degree of wedge angle change is observed for PVB sheets, which are located in the lower positions of the pallet (PVB sheets 76-80) (graph 12). The average value of the PVB wedge angle calculated after 72 hours of relaxation is $0.310 \mathrm{mrad}$ ( $\mathrm{tab} 2$ ) for the upper wedge
PVB sheets on the pallet $(0.033 \mathrm{mrad}$ reduction compared to the value immediately after cutting), 0.314 mrad (tab 3) for the medium wedge PVB sheets on the pallet (0.03 mrad reduction) and $0.326 \mathrm{mrad}$ (tab 4) for wedge PVB sheets at the bottom of the pallet (reduction by $0.014 \mathrm{mrad})$. For wedge PVB placed at the bottom of the pallet, it is necessary to consider further relaxation of the PVB after lamination, which can also lead to lamination defects. This would require further research.

The shrinkage of the wedge PVB sheets on the pallet plays an important role in changing the value of the wedge angle (graph 13). The relaxation process must be considered in the development phase, when the wedge angle of the wedge PVB cylinder is calculated.

We observe a linear correlation between the vertical shrinkage and the value of the wedge angle (graph 19). We observe a linear correlation between horizontal shrinkage and the value of the wedge angle (graph 18). Shrinkage in the vertical and horizontal directions stops after 48 hours of relaxation (tab. 5-6, graph 1415). The value of the wedge angle stops changing after 48 hours of relaxation (tab. 7 , graph 16). The recommended relaxation time of wedge PVB is 48 hours based on this examination. The relaxation of the wedge PVB sheets can significantly affect the quality of the final laminate, because the value of the wedge angle changes over time due to the relaxation of the wedge PVB sheets after cutting. The relaxation effect is well observable for wedge PVB sheets, which are located in the upper part of the pallet $+0.86 \%$ and for PVB sheets, which are located in the middle of the pallet $+0.54 \%$. The lowest degree of relaxation + $0.18 \%$ is observable for PVB sheets, which are placed on the bottom positions of the pallet. Based on the relaxation study, it is recommended to stack PVB sheets on a pallet of a maximum of 40 pieces so that the PVBs can effectively relax within 48 hours and there is no risk of further shrinkage in the subsequent lamination process (graph 13). Not only could there be a risk of lamination defects, but at the same time there could be an uncontrolled change in the value of the wedge of the PVB and thus a change in the wedge of the glass.

\section{Conclusion}

The aim of this work was to discuss how the process of processing PVB roll changes the input wedge angle and whether it is necessary to take into account changes in wedge angle caused by the process of unwinding and cutting PVB from the roll in the process of defining wedge angle input roll.

In this work, the effect of the change of wedge angle caused by unwinding and cutting PVB from the roll and its subsequent relaxation on the pallet before 
processing the PVB in the layering process is studied. It was measured that the wedge angle value changes depending on the PVB cutting technology from the roll. In this particular case, a change in the wedge angle was observed after cutting the PVB roll onto the PVB sheets, increasing the wedge angle by $0.0195 \mathrm{mrad}$. PVB relaxation took place on a pallet of 80 pieces. It was measured that the optimal relaxation time is 48 hours, as the wedge angle value was established for all positions after 48 hours.

As the PVB relaxes, the wedge angle value of the PVB changes again, depending on its position on the pallet. In the case of PVB sheets placed in the upper part on the pallet, the wedge angle value decreased after 72 hours of relaxation to $0.310 \mathrm{mrad}$, which is a wedge angle value that is 0.004 lower than the wedge angle value before PVB processing. It can therefore be stated that the PVB placed on the upper part of the pallet relaxes at least back to the original value of the wedge angle. The PVB placed on the middle part of the pallet relaxes at 0.314 , which is a value that does not differ much from the value that was measured on the PVB roll itself before processing - $0.3135 \mathrm{mrad}$, the difference is only $0.0005 \mathrm{mrad}$. The PVB placed in the middle part of the pallet relaxes at least back to the original value of the wedge angle. The PVB, which is located at the bottom of the pallet, does not relax to the original wedge angle. The wedge angle value after relaxation for a given PVB is $0.326 \mathrm{mrad}$, which is $0.0125 \mathrm{mrad}$ higher than the value on the input roll. It can therefore be concluded that the PVB relaxation for the leaves at the bottom of the pallet did not take place completely.

It is recommended to reduce the number of PVB sheets per pallet to 40 or fewer pieces in order to eliminate possible undesirable wedge angle changes that may occur during further processing of wedge PVB glass, for example in the lamination process, as PVB has not completely relaxed on the pallet and may occur an additional relaxation.

\section{Acknowledgement}

I'd like to express my deepest thanks to my colleague Tereza Kordová for her contribution in this study. I'm extremely grateful to Martin Havlik Míka for his professional guidance, advice and for his contribution in this study.

\section{References}

[1] QIN, F. C., LIN, Y. P., HUANG, H. P. D.; SHIEH; Maximal Acceptable Ghost Images for Designing a Legible Windshield-Type Vehicle Head-Up Display. IEEE Photonics Journal, 2017, vol. 9, no. 6, doi 10.1109/JPHOT.2017.2758820.
[2] BAIRD, D. G.; COLLIAS, D. I.; Polymer processing: principles and design, 2nd eddition. John Wiley \& Sons, 2014, ISBN: 978-0-470-93058-8.

[3] BAUM, E., MUGDAN, M.; Process of preparing acetaldehyde. 1914, US1096667A, https:// patents.google.com/patent/US1096667A/en.

[4] ANBANG, J., YONGSHENG, L., SHAOMING, D., CHONGJI, Y.; Plasticizer composition of polyvinyl butyral (PVB) and PVB resin composition containing same. 2013, CN102120832B, https://worldwide.espacenet.com/publicationDetails/biblio?FT $=$ D\&date $=20130508 \& D B=E P O D O C$ \&lo-

cale $=\& C C=C N \& N R=102120832 B \& K C=B \&$ $\mathrm{ND}=1$.

[5] RAUWENDAAL, C.; Polymer Extrusion. Polymer Extrusion, 5th eddition. Hanser, 2014, pp. 1-16, doi 10.3139/9781569905395.fm.

[6] HADDAD, T., SHAHEEN, B. W., NÉMETH, I.; Improving Overall Equipment Effectiveness (OEE) of Extrusion Machine Using Lean Manufacturing Approach. Manufacturing Technology, 2021, vol 21, no 1, pp. 56-64, doi 10.21062/mft.2021.006.

[7] PINKELSKY, J., ZURAVSKY, I., CERNOHLAVEK, V., CAIS, J., STERBA, J.; Influence of production technology on selected polymer properties. Manufacturing Technology, 2021, vol 21, no 4, pp. 520-530, doi 10.21062/mft.2021.051.

[8] ARNDT, M., KREMERS, S.; Composite glass having reduced thickness for a head-up display (HUD). 2019, US10350859 (B2), https://worldwide.espacenet.com/publicationDetails/biblio?FT $=$ D\&date $=20190625 \& D B=E P O D O C$ $\& C C=C A \& N R=2968724 C$.

[9] KLUCZEWSKI, W., GIER, S., LUCKE, S.; Method for producing wedge-shaped, thermoplastic films and use thereof. 2018, CN107614233 (A), https://worldwide.espacenet.com/publicationDetails/biblio?FT $=$ D\&date $=20180119 \& D B=E P O D O C$ $\& C C=C N \& N R=107614233 \mathrm{~A}$.

[10] DEPING, L, XIAOMIN, X; HUD glass product with reflecting film layer. 2016, CN105842850 (A), https://worldwide.espacenet.com/publicationDetails/biblio $? F T=$ D $\&$ date $=20160810 \& D B=E P O D O C$ $\& C C=C N \& N R=105842850 A$. 
[11] PENG, S., REN, H., CAO, X., YANG, Y.; Continuous forming of ultrathin glass by float process. Applied glass science, 2019, vol 3, no 10, pp. 275-286, doi 10.1111/ijag.13132.

[12] HURLBUT, J., CASHEN, D., ROBB, E., SPANGLER, L.; Next Generation PVB Interlayer for Improved HUD Image Clarity. SAE International journal of passenger cars - mechanical systems, 2016, vol 9, no 1, pp. 360-365, doi 10.4271/2016-01-1402.

[13] LIU, B., SUN, Y., LI, Y., WANG, Y.; Systematic experimental study on mechanical behavior of PVB (polyvinyl butyral) material under various loading conditions. Polym. Eng. Sci., 2012, vol 52 no 5, pp. 1137-1147, doi $10.1002 /$ pen. 22175 .
[14] JIN, J.; Machining process of PVB film. 2015, CN104859153 (A), https://worldwide.espacenet.com/publicationDetails/biblio?FT $=$ D\&date $=20150826 \& D B=E P O D O C$ \&lo-

cale $=\& C C=C N \& N R=104859153 A \& K C=A \&$ $\mathrm{ND}=2$.

[15] MARCUS, M. A.; Simultaneous head-up display windshield wedge-angle and layer-thickness measurements. Multilayer structure assessment, 2016 https://www.spie.org/news/6610-simultaneous-head-up-display-windshield-wedge-angle-and-layer-thickness-measurements?SSO $=1$. 